\title{
Open Midplane Designs Based on Sector Coils in Superconducting Dipoles
}

\author{
Jens Bruér and Ezio Todesco
}

\begin{abstract}
This paper presents a study of the effects of opening up the midplane in conventional sector coil dipoles, also known as $\cos \theta$-designs. The open midplane design is a candidate for the higher luminosity upgrade for the LHC, and also for the future beta beam project at CERN, which has the heat deposition mainly concentrated in the midplane of the dipoles. By opening up the midplane, the major part of the spray particles can be avoided, allowing the use of strong superconductive magnets. The aim of this study is to maintain good field quality after a gap in the midplane has been inserted. Short sample field and the electromagnetic force distribution will also be presented for some solutions.
\end{abstract}

Index Terms-Field quality, open midplane, superconducting magnets.

\section{INTRODUCTION}

$\mathbf{P}$ ARTICLE accelerators can have a significant radiation emitted on the horizontal plane for different reasons. In synchrotron light sources the acceleration needed to curve the electron trajectories give rise to synchrotron light in the horizontal plane, which is used to carry out different types of analysis and experiments. In a proton collider such as the Large Hadron Collider the synchrotron light is not significant, but the debris from the interaction points (IP) are focused on the horizontal and on the vertical plane by the inner triplet quadrupoles around the IP. For this reason, in the separation dipoles placed after the triplet a significant amount of energy is deposited in the horizontal plane. In future projects such as a beta-beam ring, the beam is undergoing a beta decay which affects the conductors close to the horizontal plane [1].

On the other hand, the most efficient way to create a vertical dipolar field in an electromagnet is to have current lines as close as possible to the horizontal plane. For superconducting magnets, the energy deposited in the coil may induce quenches and therefore limit the magnet performances. For this reason, coil lay-outs without conductor in the horizontal plane (the so-called open mid-plane design) have been proposed in the past [2]-[4]. Among these references, two works were based on a block coil [2], [4], and one on a sector coil [3]. In this work we systematically explore the feasibility of an open midplane design based on sector coils.

In Section II the constraints given by field quality are discussed and some lay-outs are proposed; in Section III we analyze the short sample field given by these lay-outs, and in

Manuscript received August 18, 2008. First published May 27, 2009; current version published July 15, 2009.

The authors are with the Accelerator Technology Department, CERN, 1211 CH, Switzerland (e-mail: jens.erik.bruer@cern.ch; ezio.todesco@cern.ch).

Color versions of one or more of the figures in this paper are available online at http://ieeexplore.ieee.org.

Digital Object Identifier 10.1109/TASC.2009.2018513

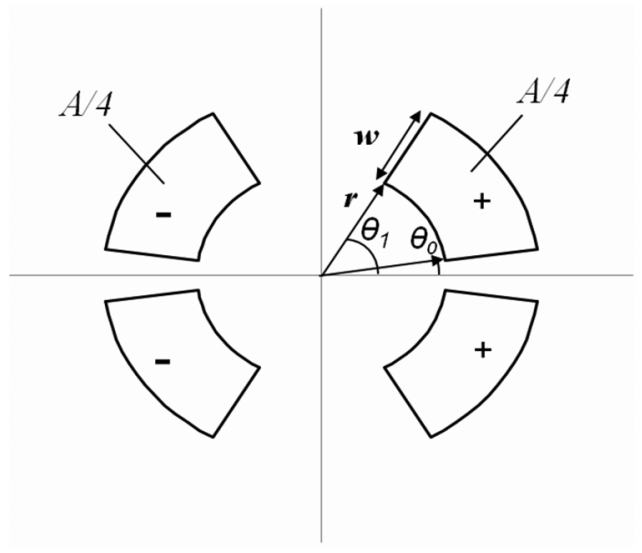

Fig. 1. Cross section of a one-sector coil with an open midplane.

Section IV we evaluate the electromagnetic forces acting on the coils during the magnet powering.

\section{MAGNeTIC FIELD QuALITY}

\section{A. Equations for Sector Coils}

The magnetic field is expanded in the usual power series:

$$
B(z)=\sum_{n=1}^{\infty} C_{n}\left(\frac{z}{R}\right)^{n-1}=\sum_{n=1}^{\infty}\left(B_{n}+i A_{n}\right)\left(\frac{z}{R}\right)^{n-1}
$$

For the specific case of a four-fold symmetric coil layout, as shown in Fig. 1, the skew multipoles $A_{n}$ and the even normal $B_{2 n}$ cancel out. In a dipole one aims at $B(z)=B_{1}$, i.e., the higher order multipoles should be set to zero. The equation for the multipoles for a one sector with open midplane as shown in Fig. 1 reads:

$$
B_{n}=-2 \frac{\mu_{0}}{2 \pi} R^{n-1} \int_{r}^{r+w} \int_{\theta_{0}}^{\theta_{1}} j \rho^{-n} \cos (n \theta) \rho d \rho d \theta,
$$

which gives

$$
\begin{aligned}
B_{n}=-2 \frac{\mu_{0} j R^{n-1}}{\pi(2-n) n} & \left((r+w)^{2-n}-r^{2-n}\right) \\
& \times\left(\sin \left(n \theta_{1}\right)-\sin \left(n \theta_{0}\right)\right), \quad n \neq 2
\end{aligned}
$$

With one-sector per quadrant, one can only set one multipole to zero, i.e., $B_{3}$. The trivial solution is

$$
\theta_{1}=\frac{\pi}{3}-\theta_{0}
$$

For instance, a $5^{\circ}$ opening implies a $55^{\circ}$ pole angle to cancel $B_{3}$. By adding copper wedges to the coil, additional sectors are 




Fig. 2. A three sector coil with an open midplane.

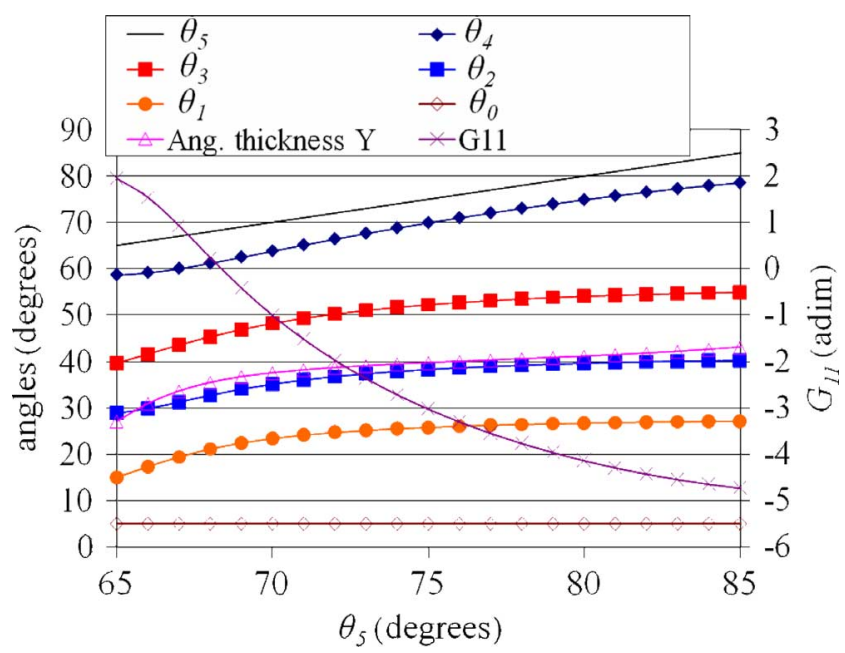

Fig. 3. Angle configurations for the three sector coil shown in Fig. 2 cancelling $B_{3}$ to $B_{9}$, with a $5^{\circ}$ opening in the midplane.

formed (see Fig. 2) and more sin functions appear in the angular part of (3). One can then cancel out more multipoles. The magnitude of the multipoles is decreasing with order, so the highest priority is to cancel out the low order multipoles, typically $B_{3}$ to $B_{11}$. This level of optimization is one of the possible choices, which strongly depends on the position of the magnet in the lattice and on the type of machine.

Two sectors allow canceling three multipoles, namely $B_{3}$ to $B_{7}$. To cancel out the first five multipoles $\left(B_{3}=B_{5}=B_{7}=\right.$ $\left.B_{9}=B_{11}=0\right)$, we need at least three sectors, giving the following system of equations:

$$
\begin{aligned}
G_{n} \equiv & \sin \left(n \theta_{5}\right)-\sin \left(n \theta_{4}\right)+\sin \left(n \theta_{3}\right)-\sin \left(n \theta_{2}\right) \\
& +\sin \left(n \theta_{1}\right)-\sin \left(n \theta_{0}\right)=0 \\
n= & 3,5,7,9,11
\end{aligned}
$$

To find solutions to this system of non-linear equations, numerical methods were used. A fine grid with a step of $1^{\circ}$ over the five variables has been considered; then the best solutions have been refined using a Newton method [5].

\section{B. Solutions for Sector Coils}

The one-parameter family of solutions cancelling the first four multipoles for a three sector coil is shown in Fig. 3 for a $5^{\circ}$



Fig. 4. The sector sizes versus the size of the opening, for three sector coils.

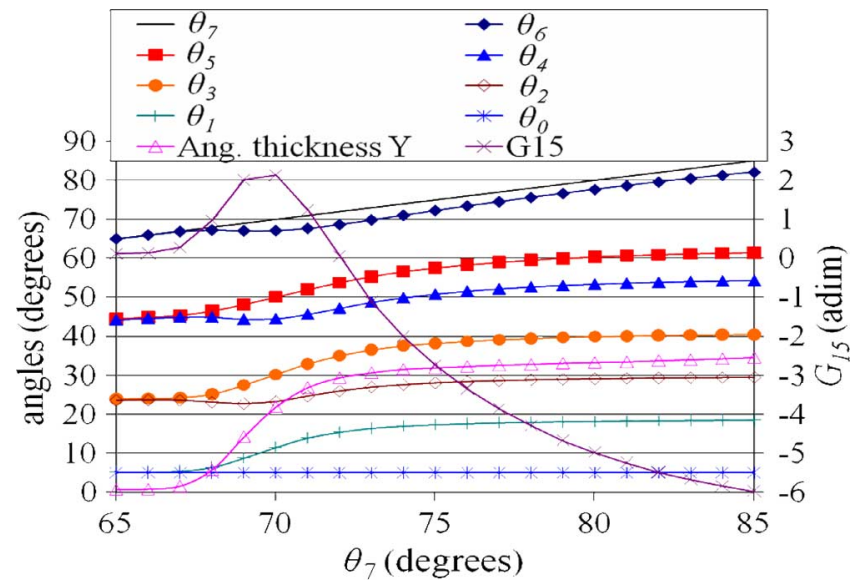

Fig. 5. Solutions for canceling out $B_{3}$ to $B_{13}$, for a four sector coil with a $5^{\circ}$ opening.

opening; we also plot the angular function $G_{11}$, which is equal to zero for $\theta_{5} \sim 68^{\circ}$. We also give $Y$, which is the sum of the angular thickness of the three sectors;

$$
Y=\vartheta_{5}-\vartheta_{4}+\vartheta_{3}-\vartheta_{2}+\vartheta_{1}-\vartheta_{0}
$$

it ranges between $30^{\circ}$ and $45^{\circ}$ : this means that the fraction of the coil covered by wedges will be much larger with respect to the no opening case, where the angular thickness $Y$ is around $60^{\circ}$. These solutions are independent of the magnet aperture and of the coil width. If we repeat the same estimate for different angular openings, it turns out that for three sector coil the total angular thickness of the coil $Y$ decreases for larger openings, and goes to zero for an opening of $13^{\circ}$ (see Fig. 4).

The three sector coil is an interesting model to understand the main feature of the field quality optimization with open midplane. Indeed, in many cases three sectors do not provide enough free parameters to optimize field quality. With a four sector coil we can cancel $B_{3}$ to $B_{13}$ and we still have one free parameter. In Fig. 5 we plot this one parameter family as a function of the pole angle $\theta_{7}$, for a $5^{\circ}$ opening. The interesting region is between $\theta_{7}=70^{\circ}$ and $\theta_{7}=75^{\circ}$, which combines a reasonable coil area (total angular thickness $Y$ is around $30^{\circ}$ ) and a pole angle that is not too large. By relaxing the condition to cancel 


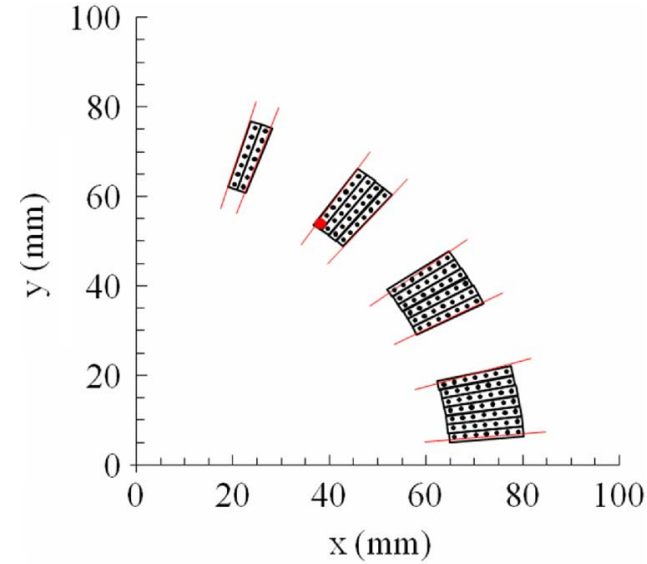

Fig. 6. Cross section of a four sector coil with a $5^{\circ}$ opening with an LHC cable, solution computed for sector coils (radial red lines), and peak field location (red marker).

out $B_{13}$, one gets a two parameter family of solutions with a four sector coil, which provides better flexibility and a slightly larger coil area.

\section{Coil Layouts With Rutherford Cable}

When the coil is built with Rutherford cable, the actual thickness of the cable imposes an additional constraint. The coil will not exactly match the analytical solution, thus producing non-negligible multipoles. In principle this can be corrected by slightly moving and tilting the sectors. In this section we explore this possibility, using four different cables (Tevatron [6], HERA [7], RHIC [8] and LHC [9] main dipoles) combined with three different aperture radiuses (40, 50 and $60 \mathrm{~mm}$ ), resulting in 12 different cases. The size of the opening has been set to $5^{\circ}$ and $10^{\circ}$.

We found that for the three sector coils based on the previous computations we did not manage to correct the multipoles due to the fact that the sector thickness is a discrete variable, i.e. must be an integer multiple of the cable thickness. On the other hand, in the case of four sector coils with a $5^{\circ}$ opening, solutions with good field quality (normalized multipoles $\left|b_{3}\right|$ to $\left|b_{11}\right|<1$ at a reference radius $R=2 / 3 r$ ) were found for each of the 12 cases (see Fig. 6 for a $65 \mathrm{~mm}$ aperture radius with a LHC MB cable [9]).

When the opening is increased to $10^{\circ}$, only in a few cases we were able to adjust the sector coil solutions made with Rutherford cable to generate good field quality. In this case we considered a two layer coil, with three sectors in the inner layer and two sectors in the outer one. This provides additional parameters to tune the multipoles, plus the added benefit of a larger coil area, thus increasing the field. For the two layer, five sector coil layout, lay-outs were found for each of the 12 combinations of cable and aperture radius (see Fig. 7).

\section{SHORT SAMPLE FIELD}

The maximum obtainable field $B_{\mathrm{SS}}$ in the center of the dipole is denoted short sample field, and it is the central field when the current density in the coil is equal to the critical current density. The short sample field is in other words the limit of the performance of the magnet.

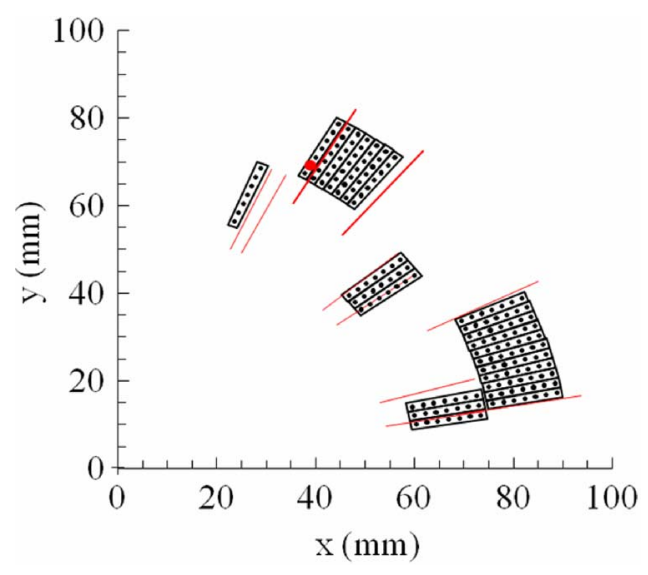

Fig. 7. Cross section of a five sector coil, two layers, with a $10^{\circ}$ opening with an LHC cable, solution computed for sector coils (radial red lines), and peak field location (red marker).



Fig. 8. Short sample field for layouts with aperture radius of $60 \mathrm{~mm}$ (markers), versus coil width, and $60^{\circ}$ sector coil reference values. $\mathrm{Nb}-\mathrm{Ti}$ at $1.9 \mathrm{~K}$.

Here we are neglecting the contribution of the iron yoke, and we consider the case of Nb-Ti at $1.9 \mathrm{~K}$. We compute $B_{\mathrm{Ss}}$ for the $60^{\circ}$ sector coil without opening, and for the $5^{\circ}$ and $10^{\circ}$ opening using four different cables, with an aperture radius of $60 \mathrm{~mm}$. With a $5^{\circ}$ opening the short sample field is reduced from $7.5 \mathrm{~T}$ to $6 \mathrm{~T}$ in the case of $15 \mathrm{~mm}$ width and from $6 \mathrm{~T}$ to $4 \mathrm{~T}$ for a $10 \mathrm{~mm}$ width. A larger reduction is found for larger openings as expected: for instance, with a $30 \mathrm{~mm}$ cable width and a $10^{\circ}$ opening the field decreases from $10 \mathrm{~T}$ to $6 \mathrm{~T}$ (see Fig. 8).

Indeed, this comparison is not completely fair since the layouts with open midplane use much less superconducting material than the standard ones. A more fair comparison is to plot $B_{s s}$ versus the coil area. Since the area is a quantity that is difficult to appreciate, we rather use the equivalent coil width

$$
w_{e q}=r\left(\sqrt{1+\frac{3 A}{2 \pi r^{2}}}-1\right),
$$

i.e. the width of the $60^{\circ}$ layer with the same coil area [10]. Results are shown in Fig. 9: if the comparison is carried out on the same coil area, the loss given by an open midplane lay-out is in general a few percent for $5^{\circ}$, and up to $10 \%$ for $10^{\circ}$. 




Fig. 9. Short sample field for lay-outs with aperture radius of $60 \mathrm{~mm}$ (markers), versus coil area (equivalent coil width), and $60^{\circ}$ sector coil reference values. $\mathrm{Nb}-\mathrm{Ti}$ at $1.9 \mathrm{~K}$.



Fig. 10. Electromagnetic forces for a one layer sector coil with a $5^{\circ}$ opening (left) and for a two layer sector coil with a $10^{\circ}$ opening (right).

\section{ELECTROMAGNETIC FORCES}

In $\cos \theta$ dipoles based on the sector layouts, the sum of the azimuthal component of the electromagnetic force on each sector results in a pressure on the midplane. In an open midplane design, this would mean that a wedge has to be inserted in the gap, to hold the conductors. The proposed solutions for the one layer cases have a force distribution very similar to a standard $\cos \theta$ dipole (see Fig. 10, calculation with Roxie [11]); the wedge in the midplane could carry inside a cooling structure.

For the two layer design, in all cases the force in the inner layer block closer to the midplane is only radial. Moreover, the azimuthal forces on the midplane resulting from the upper blocks are small. This means that the inner layer needs less support on the midplane, where one can use some space for a cooling channel.

\section{CONCLUSIONS}

We analyzed the field quality constraints for an accelerator dipole coil (i.e. a field homogeneity of $10^{-4}$ over at least $2 / 3$ of the aperture) based on the $\cos \theta$ lay-out with an open midplane. We first considered a simplified model based on a sector coil, showing that solutions with one layer and three or four sectors can be found. An opening of $5^{\circ}$ to $10^{\circ}$ is possible, whereas larger openings give rise to very sparse coils, or to no solution at all. We then considered how to design a $5^{\circ}$ or $10^{\circ}$ open midplane coil with a Rutherford cable; in this case the sector solutions, used as first guess, need a fine tuning. Four sectors are needed to have enough free parameters to carry out the field quality optimization. A solution with two layers and five blocks has also been presented.

The short sample field of the open midplane coils is lower than in the case of no opening and same aperture and coil width by $20-30 \%$ for $5^{\circ}$ opening and by $40-50 \%$ for $10^{\circ}$ opening. Indeed, the open midplane lay-outs have a much smaller coil area. When the comparison is made between lay-outs with the same quantity of cable, the open midplane cases give a short sample field which is only $5-10 \%$ lower than the standard case.

The advantage of an open midplane design with respect to a larger aperture standard design, such as proposed in [1], containing a shielding with a channel removing heat has to be evaluated case-by-case. This choice depends on the main magnet parameters (field, aperture, total size) and on the type and on the energy spectrum of the particles spraying on the midplane.

\section{REFERENCES}

[1] C. Vollinger and E. Wildner, A Large Aperture Superconducting Dipole for Beta Beams to Minimize Heat Deposition in the Coil, CERN AT 2007-21 (MCS), 2007.

[2] R. Gupta, "Optimization of open midplane dipole design for LHC IR upgrade," in Particle Accelerator Conference, 2003, p. 3055.

[3] N. V. Mokhov et al., "Energy deposition limits in a Nb3Sn separation dipole in front of the LHC high-luminosity inner triplet," in Particle Accelerator Conference, 2003, p. 1748.

[4] P. McIntyre and A. Sattarov, "Towards an optimization of the LHC intersection region using new magnet technology," in Particle Accelerator Conference, 2005, p. 2920.

[5] J. Bruer, thesis.

[6] R. Hanft et al., Magnetic field properties of Fermilab Energy Saver Dipoles TM-1182, 1630, 03/1983.

[7] S. Wolff, "The superconducting magnet system for HERA," in Proceedings of MT19, C. Marinucci and P. Waymuth, Eds., Zurich, 1995, SIN.

[8] M. Anerella et al., "The RHIC magnet system," Nucl. Instrum. Meth., vol. A499, pp. 280-315, 2003.

[9] L. Rossi, "The LHC main dipoles and quadrupoles towards series fabrication," IEEE Trans. Appl. Supercond., vol. 13, pp. 1221-1228, 2003, and 3875-6.

[10] L. Rossi and E. Todesco, "Electromagnetic design of superconducting dipoles based on sector coils," Physical Review Special Topics-Accelerators and Beams, vol. 10, p. 112401, 2007.

[11] S. Russenschuck, CERN 99-02, 1999. 